\title{
种学通极
}

\section{纳米 $\mathrm{Fe}$ 的低温比热 ${ }^{*}$}

雒建林 白海洋 金 铎

(中国科学院低温技术实验中心, 北京 100080)
孙继荣

(中国科学院物理研究所, 北京 100080)

\section{关键词纳米材料 低温比热}

纳米微晶是由纳米量级 $(1 \sim 100 \mathrm{~nm})$ 晶粒构成的多晶物质. 纳米微晶是由两种组元组 成, 其一为晶体组元, 该组元中所有原子都位于晶格内的格点上; 另一部分为界面组元, 其原 子都位于晶粒之间的界面上 [1]. 由于纳米微晶物质的界面比大尺寸晶粒物质大得多, 且晶体 组元又远小于大尺寸晶粒物质, 这样纳米微晶就表现出不同于普通晶体的结构特点和物理性 质. 纳米铁的磁性和 Mössbauer 谱的测量 ${ }^{[2,3]}$ 表明, 纳米 $\mathrm{Fe}$ 的饱和磁化强度远小于大块 $\alpha-\mathrm{Fe}$ 和非晶铁的饱和磁化强度, 并且纳米 $\mathrm{Fe}$ 界面组分的居里温度 $T_{\mathrm{c}}$ 比大块多晶 $\mathrm{Fe}$ 低, 这意味着 在纳米铁中可能存在一种新的磁结构; 不同粒度和压力下的纳米 $\mathrm{Ni}, \mathrm{Fe}$ 等的电导率 ${ }^{[4] 1)}$, 测量 表明, 纳米材料的电导率比普通晶体低一二个数量级, 且和界面的物理和化学状态关系很大. 此外, 自扩散系数 ${ }^{[9]}$ 、弹性模量 ${ }^{[0}$ 等物性的测量研究也表明纳米材料和大块样品有很大的差别.

低温比热测量是研究固体的电子能态结构、相变、界面结构、晶体的晶格振动状态等很有 效的手段之一, 对纳米晶的低温比热测量 ${ }^{[7,8]}$ 也出现不同于大块晶体的性质, 主要表现为比热 值增大. 目前这方面的工作还不多, 测量温区也比较高 ( $100 \mathrm{~K}$ 以上), 实验结果还不够详尽和 系统, 许多本征的问题还不清楚. 本文报道了纳米 $\mathrm{Fe}$ 的 $1 \sim 25 \mathrm{~K}$ 温区的低温比热的测量结 果, 发现比热值在 $T<16 \mathrm{~K}$ 可用 $\gamma T+\alpha T^{2}+\beta T^{3}$ 拟合, 其中 $T^{2}$ 项的出现可以归因于表面声子 谱的贡献; 电子比热系数 $\gamma$ 比大块 $\alpha-\mathrm{Fe}$ 小一倍, 可能与电子能级间距变大有关; 在较高温 区, 纳米 $\mathrm{Fe}$ 的比热明显高于大块 $\alpha-\mathrm{Fe}$ 的比热, 可能存在晶格振动 Einstein 模.

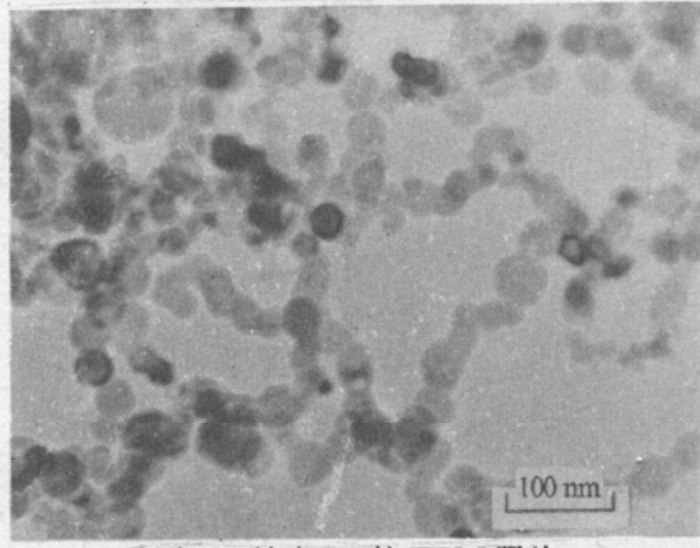

图 1 纳米 $\mathrm{Fe}$ 的 TEM 照片

\section{1 实验方法}

纳米晶 $\mathrm{Fe}$ 的制备过程首先使用纯度在 $99.999 \%$ 以上的块材 $\alpha-\mathrm{Fe}$ 作为原料, 利用气 态蒸溜法得到纳米量级的超细粉末, 然后加 压 $4 \times 10^{6} \mathrm{kPa}$ 压成直径 $8 \mathrm{~mm}$ 厚约 $2 \mathrm{~mm}$ 的圆 片 (参见文献 [4] ${ }^{1)}$ ). TEM 的分析结果表明 大部分颗粒为球状, 且平均粒度为 $40 \mathrm{~mm}$ (见 图 1). X-ray 衍射实验表明存在不超过界面 部分 $8 \%$ 的 $\mathrm{Fe}_{3} \mathrm{O}_{4}$.

1994-10-18 收稿, 1995-06-20 收修改稿

*国家自然科学基金、国家科学技术委员会和中国科学院专项基金资助项目

1) Sun J, Yi S. Resistance of naaocrystalline iron. to published 
比热测量使用标准绝热量热法, $4.2 \mathrm{~K}$ 以下的温区由 $\mathrm{He}^{4}$ 小液池减压降温得到. 对高纯 $\mathrm{Cu}$ 标准样品的测量数据表明此系统的测量误差小于 $2 \%$.

\section{2 结果及分析}

纳米 $\mathrm{Fe}$ 样品在 $1 \sim 25 \mathrm{~K}$ 的低温比热曲线如图 2 所示. 比热的数值随温度的升高而增 大, 当温度高于 $16 \mathrm{~K}$ 时, 比热上升变陡. 为了和大块 $\alpha-\mathrm{Fe}$ 样品比较, 图中的实线给出了大块 $\alpha-\mathrm{Fe}$ 的比热数据 ${ }^{[9]}$. 在低温下两者比热不重合, 随着温度的升高, 纳米唱 $\mathrm{Fe}$ 的比热比大块 $\mathrm{Fe}$ 的比热上升的快, 其差别越来越显著.

为了分析低温区的数据, 图 3 给出了纳米 $\mathrm{Fe}$ 和大块铁在 $1 \sim 16 \mathrm{~K}$ 之间的 $C / T-T$ 关系 图. 纳米铁的比热数据可以用 (1) 式拟合:

$$
C=\gamma T+\alpha T^{2}+\beta T^{3},
$$

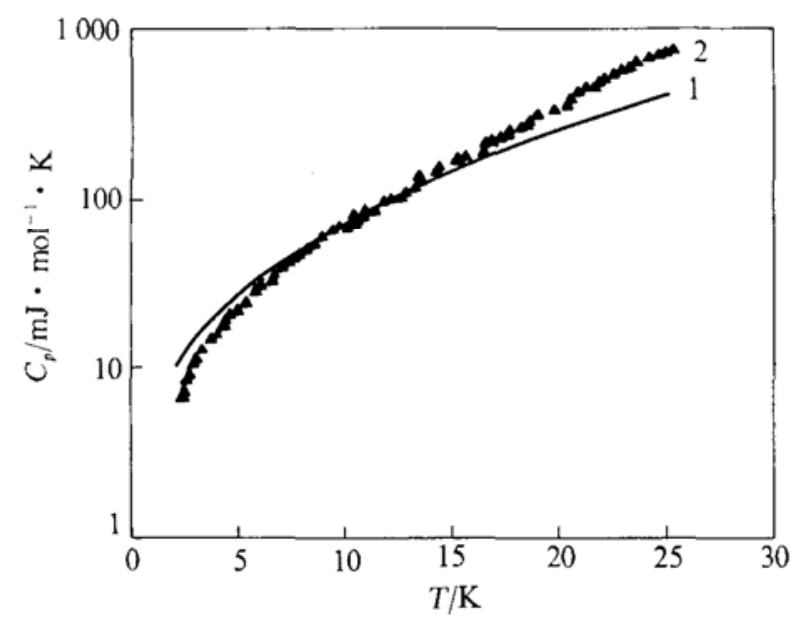

图 2 纳米 $\mathrm{Fe}$ 在 $1.8 \sim 26 \mathrm{~K}$ 的比热图 1 为大块 $\mathrm{Fe}$ 样品, 2 为纳米 $\mathrm{Fe}$ 样品

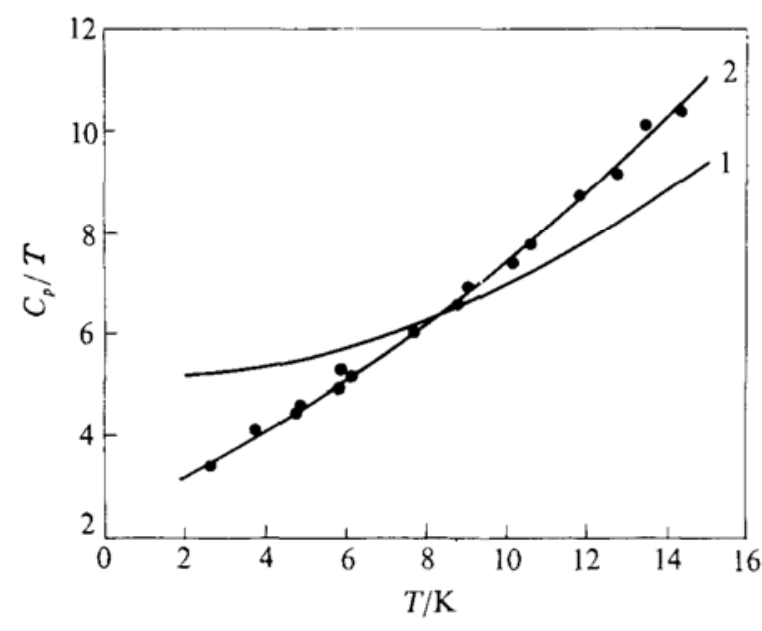

图 $3 C p / T$ 随温度 $T$ 变化曲线 1 为大块 $\mathrm{Fe}$ 样品, 2 为纳米 $\mathrm{Fe}$ 样品

图中的实线即为拟合曲线. 表 1 列出了这几项的拟合系数和大块 $\alpha-\mathrm{Fe}$ 的差异. 可见, 在低温区 $(T<16 \mathrm{~K})$, 和大块 $\mathrm{Fe}$ 比较, 纳米唱 $\mathrm{Fe}$ 的电子比热系数减小了近一倍 ( $\gamma$ 值); 晶格比 热变化不大 ( $T^{3}$ 项) ; 且出现了比较大的 $T^{2}$ 项. 纳米微唱材料由晶体组元和界面组元组成, 由 于大量的表面和界面的存在, 则出现了声子谱激发的表面模式, 使得声子谱软化, 其对比热的 贡献可以由下式表示 ${ }^{[10]}$ :

$$
C=B S T^{2},
$$

表 1 纳米 $\mathrm{Fe}$ 和大块 $\alpha-\mathrm{Fe}$ 的比热比较 ${ }^{\text {a) }}$

\begin{tabular}{cccc}
\hline & $\gamma\left(\mathrm{mJ} / \mathrm{mol} \cdot \mathrm{K}^{2}\right)$ & $\alpha\left(\mathrm{mJ} / \mathrm{mol} \cdot \mathrm{K}^{3}\right)$ & $\beta\left(\mathrm{mJ} / \mathrm{mol} \cdot \mathrm{K}^{4}\right)$ \\
\hline 纳米 $\mathrm{Fe}$ & 2.39 & 0.36 & 0.014 \\
大块 $\mathrm{Fe}$ & 4.98 & 0 & 0.019 \\
\hline
\end{tabular}

a) $\alpha-\mathrm{Fe}$ 的比热参数取自文献 [9], $\gamma$ 是电子比热系数, $\alpha$ 为 $T^{2}$ 项系数, $\beta$ 为 $T^{3}$ 项系数

$S$ 是表面积, $B$ 为常数. 对普通的大块多晶或单晶材料, 和纳米晶相比较, 表面和界面所占比 
例非常小, 所以表面声子模的贡献不能被实验观察到. 对我们所测量的纳米微唱 $\mathrm{Fe}$, 平均粒 度 $40 \mathrm{~nm}$, 界面厚约 2 4 nm, 粗略估计界面组元占整个样品的四分之一左右, 这样声了表面 模对比热的贡献 $T^{2}$ 项就比较显著的表现出来, 在 $2 \mathrm{~K}$ 时约占整个比热的 $23 \% . T^{3}$ 项是唱体 组无中晶格振动对比热的贡献, 满足 Debye 规律, 对于低温区的纳米铁样品, 这一项变化不人.

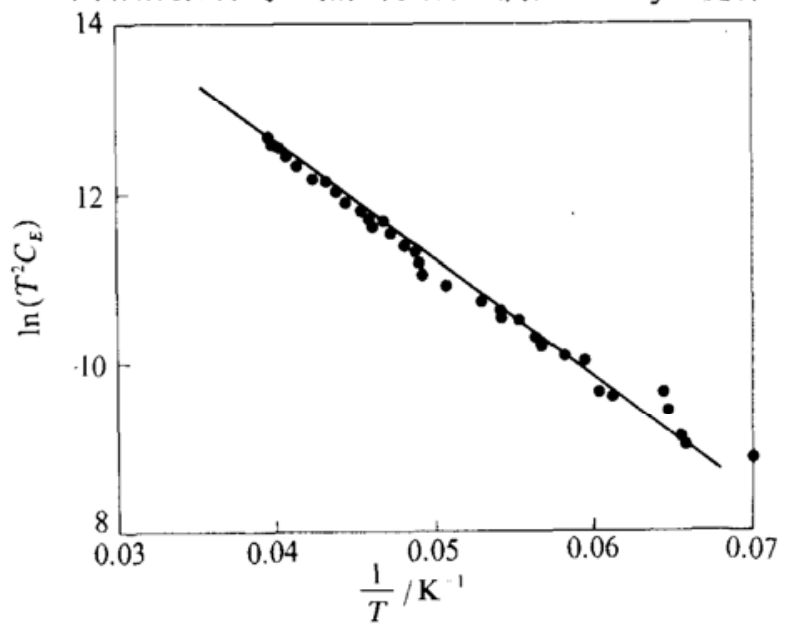

图 $4 T^{2} C_{E}$ 随温度倒数变化曲线

由表 1 我们得到另一个非常重要的缩 果是纳米 $\mathrm{Fe}$ 的电子比热系数 $\gamma$ 比大块 $\alpha-\mathrm{Fe}$ 减小近一倍. 电子比热系数 $\gamma$ 和费米面上 的电子结构 (浓度、态密度等) 有直接的关 系, 是表征电子运动状态的物理量之一. 对 于我们所测量的纳米 $\mathrm{Fe}$ 样品, 对其电子比 热的影响主要来自于以下几个方面的贡 献: 其一, 由于界面组元在整个样品中占有 很大的比例, 处于界面原子上的电子基 本被束缚在界面上, 同时, X-ray 分析结果 也表明,有少量的界面组元被氧化成 $\mathrm{Fe}_{3} \mathrm{O}_{4}$ 而成为绝缘体 $(<8 \%)$. 于是我们叮以考意 一个极端的情况, 认为处于界面原子上的壳层电子完全被局域, 不参与对电子比热的页献, 参 与热运动的载流子只限于晶体组元. 由 $\mathrm{L}$ 面的估计, 对于我们的纳米 $\mathrm{Fe}$ 样品, 和大块 $\alpha-\mathrm{Fe}$ 比较, 载流子数月减少约四分之一, 从而由于表面电子束缚态而使电子比热系数 $\gamma$ 值减小不超 过四分之一. 其二, 电子比热和费米面上的态密度成正比, 而态密度和电子能级间距的大小 有关. 电子能级间距变宽, 态密度减小, 从而电子比热减小。电子比热随能级间距 $\delta$ 的变化规 律和具体的能级分布函数有关, 并和每个粒子所包含的电子数的奇偶性有关 ${ }^{[11]}$, 电子能级间距 可以由下式估计：

$$
\delta \approx E_{f} / N=\propto V^{-1},
$$

$E_{f}$ 是 Fermi 能级, $N$ 是传导电子数, $V$ 是颗粒的体积. 随着颗粒尺寸的减小, 同时使传导电子 的数目也减小, 这样 Fermi 面上被填充的电子态数目减小, 电子能级宽度增大, 电子比热减 小. 对于我们所测量的纳米 $\mathrm{Fe}$ 样品, 和大块铁相比较, 颗粒尺小 $V$ 远小于大块 $\alpha-\mathrm{Fe}$; 这样引 起了能级间距的变化从而使得电子比热项减小, 可能是 $\gamma$ 值变小的另一个原因. 第三, 由于表 面势的影响, 使得处于表面上的传导电子的波函数产生相移, 这样对电子比热的贡献使得电 子比热系数变大 ${ }^{[12]}$. 以上三种机制都可能对纳米铁的低温电子比热有贡献, 和大块 $\alpha-\mathrm{Fe}$ 比 较, 前两个原因使得纳米铁的电子比热系数 $\gamma$ 值减小, 后一个方面使得 $\gamma$ 值增任. 我们的实验 结果表明, 纳米 $\mathrm{Fe}$ 的 $\gamma$ 值比大块 $\mathrm{Fe}$ 样品减小了约一半, 而第一项由于表面电子局域态而使 $\gamma$ 值减小不超过四分之一, 第三项表面传导电子波函数相移使得 $\gamma$ 值增大, 因此, 由于电子能级 间距变大的量子尺寸效应对纳米铁的电子比热有贡献, 在实验误差内, 其结果使电子比热系 数比大块 $\mathrm{Fe}$ 样品减小超过四分之一. 也就是说, 量子小寸效应是使电子比热变小的… 主 要原因.

纳米铁在较高温区的比热值 $(T>16 \mathrm{~K})$ 随温度上升很陡, 明显高于大块铁的比热, 高温 $区$ 的比热主要来自于唱格振动的贡献. 对于我们所测量的纳米 $\mathrm{Fe}$ 材料, 唱格振动谱已不能用 
简单的 Debye 模型来描述, 晶格比热数据已不能用简单的 $T^{3}$ 次方拟合. 在这样小的粒度下, 每 个颗粒只包含几千到几万个原子, 唱格振动的色散关系已不能用连续谱表示, 而可能出现分 立的 Einstein 模式. 对 Einstein 模型, 认为每个格点上的振动是独立的、同频率的振动, 设振 动频率为 $V_{E}$, 对应的特征温度 $T_{E}=h V_{E} / K$, 当 $T<T_{E}$ 时, 比热随温度的关系为 ${ }^{[13]}$ :

$$
C=A / T^{2} \exp \left(-T_{E} / T\right) \text {. }
$$

我们比热数据的拟合结果也表明, 如图 4 所示存在 (4) 式这一指数项的贡献. 可见在纳米 $\mathrm{Fe}$ 的唱格比热中, 存在 Einstein 模, 导致了比值随温度数值的显著增加.

在本文的数据分析中, 没有考虑 $\mathrm{Fe}$ 的铁磁有序对比热的贡献, 这是由于铁的铁磁有序转 变温度 $T_{\mathrm{C}}=1007 \mathrm{~K}$, 远高于我们比热测量温区的温度. 分析、计算的结果表明, 铁磁有序对比 热的贡献远小于电子和唱格项对比热的贡献, 在实验的误差之内.

本文首次报告了纳米尺寸 $\mathrm{Fe}$ 样品的低温比热的测量结果, 其数据分析结果表明纳米 $\mathrm{Fe}$ 和大块 $\mathrm{Fe}$ 在这一温区的比热有很大差别, 首先, 比热数值中出现了很大 $T^{2}$ 项, 这来源于表面 声子模的贡献; 其次, 纳米 $\mathrm{Fe}$ 的电子比热系数比大块 $\alpha-\mathrm{Fe}$ 小一倍, 可能存在电子能级变宽的 影响; 最后, 晶格振动谱中出现了 Einstein 模, 导致了晶格比热的显著增加.

\section{参考 文献}

I Birringer R, Gleiter H, Kein H P et al. Nanocrystalline materials an approach to a novel solid structure with gas-like disorder? Phys Lett, 1984, 102A (8): $365 \sim 369$

2 Birringer R, Holfler H, Karch J et al. Diffusion and defect data. Trans Tech Aedermannsdorf, 1988, 17

3 Herr U, Jing J, Birringer $\mathrm{R}$ et al. Investigation of nanocrystalline iron materials by Mössbauer spectroscopy. Appl Phys Lett, 1987, 50: 472 474

4 Sun Jirong, Zhang Yingzi, Rao Guanghui et al. Resistance behaviour of nanocrystal nickel. J Mater Sci Technol, 1993, 9: $185 \sim 189$

5 Horrth J, Birringer R, Gleiter H. Diffusion in nanocrystalline material. Solid State Comm, 1987, 62: 319 322

6 Schefer H E, Murschum R, Birringer R et al. Nanometre-sized solids, their structure and properties. J Less-Common Materils, 1988, 140: 161 169

7 Rupp J, Birringer R. Enhanced specific-heat-capacity $(\mathrm{Cp})$ measurements $(150 \sim 300 \mathrm{~K})$ of nanometer-sized crystalline materials. Phys Rev B, 1987, 36(15): $7888 \sim 7890$

8 Hellstem E, Fecht $\mathrm{H} \mathrm{J}$. Structural and thermodynamic properties of heavily mechanically deformed Ru and AlRu. J Appl Phys, 1989, 65: 305 310

9 Johnson V J. Properties of Materials at Low Temperature (Phase 1). Oxford: Pergamon Press, 1961, 4. 181

10 Gopal E S R. Specific Heat at Low Temperatures. New York: Plenum Press, 1966. 190

11 Stewart G R. Size effect in the electronic heat capacity of small platinum particles embedded in silica. Phys Rev B, 1977, 15(2): 1143 1150

12 Kenner V E, Allen R E. Surface contribution to electronic density of states, heat capacity and spin susceptibility. Phys Rev B, 1975, 11(8): $2858 \sim 2859$

13 Gopal E S P. Specific Heat at Low Temperatures. New York: Plenum Press, 1966. 25 